\title{
Contribuições das práticas e estágios no curso de enfermagem para a formação acadêmica
}

\author{
Contributions of practices and internships in the nursing course for academic training
}
Contribuciones de las prácticas y pasantías en el curso de enfermería para la formación académica

Suellen Hoffmann Jardim ${ }^{1 *}$, Paloma dos Santos Bernardino ${ }^{1}$, Beatriz Neres Ferreira ${ }^{1}$, Pamella Cacciari'.

\section{RESUMO}

Objetivo: A proposta do estudo é mencionar a experiência de acadêmicas de enfermagem durante as práticas e estágios realizados no decorrer do curso. Relato de experiência: Trata-se de um estudo descritivo, tipo relato de experiência, elaborado a partir das vivências das autoras do presente trabalho. Os dados obtidos na experiência das alunas evidenciaram que as práticas e estágios contribuem para o conhecimento do aluno acerca do SUS e fornece a possibilidade de vivenciar acontecimentos reais e fictícios, como realizar curativo, cuidar de um paciente com distúrbios psiquiátricos e dependência química, visita domiciliar e atender puérpera e recém-nascido, desenvolvendo assim a capacidade de analisar e responder à situações do ser enfermeiro, devido a utilização de todo o seu conhecimento adquirido em âmbito teórico e prático. Contudo, defronta-se com inseguranças, distinção entre teoria e prática e cobranças excessivas. Considerações finais: As práticas e estágios são relevantes para a formação integral do aluno de enfermagem para atuar nos diferentes níveis de atenção.

Palavras-chave: Estudantes, Conhecimento, Enfermagem, Ensino, Estágios.

\begin{abstract}
Objective: The purpose is mention the experience of nursing students during the practices and internships performed in nurse degree. Experience report: This a descriptive study, with a experience report elaborated from the experiences of the authors. The captured data in the students' experience showed that the practices and internships contribute to the student's knowledge about SUS and provides the possibility to experience real and fictitious events, such as dressing, caring for a patient with psychiatric disorders and chemical dependency, home visit and assisting puerperal and newborn babies. Thus thus developing the ability to analyze and respond to situations of being a nurse, due to the use of all their knowledge acquired in theoretical and practical scope. However, in the face of insecurities, a distinction between theory and practice and excessive demands. Final considerations: Practices and internships are relevant to the comprehensive education of nursing students to work at different levels of care.
\end{abstract}

Keywords: Students, Knowledge, Nursing, Teaching, Internships.

\section{RESUMEN}

Objetivo: El propósito es mencionar la experiencia de estudiantes de enfermería durante las prácticas y pasantías realizadas en el grado de enfermera. Informe de experiencia: El estudio descriptivo, del tipo informe de experiencia, elaborado a partir de las experiencias de los autores. Los datos obtenidos en la experiencia de los estudiantes se demostraron que las prácticas y pasantías contribuyen al conocimiento del estudiante sobre el SUS y proporciona la posibilidad de experimentar eventos reales y ficticios, como vestirse, cuidar a un paciente con trastornos psiquiátricos y dependencia química, visitas domiciliarias y asistir a bebés puerperales y recién nacidos. Así se desarrolla la capacidad de analizar y responder a situaciones de ser enfermero, debido al uso de todos sus conocimientos adquiridos en el ámbito teórico y práctico. Sin embargo, frente a las inseguridades, una distinción entre teoría y práctica y demandas excesivas. Consideraciones finales: Las prácticas y pasantías son relevantes para la educación integral de los estudiantes de enfermería para trabajar en diferentes niveles de atención.

Palabras clave: Estudiantes, Conocimiento, Enfermería, Enseñanza, Prácticas.

${ }^{1}$ Universidade do Oeste Paulista (UNOESTE), Presidente Prudente - SP.
${ }^{*}$ E-mail: suellenhoffmannjardim@hotmail.com 


\section{INTRODUÇÃO}

O enfermeiro é um integrante da equipe de enfermagem, assim, deve ter formação acadêmica para que adquira conhecimentos científicos, habilidades técnicas, raciocínio crítico-reflexivo, atitudes que acompanhem as mudanças em todos os âmbitos e consiga sanar as necessidades individuais e coletivas da equipe de enfermagem e do usuário (XIMENES NETO FRG, et al., 2020).

No cenário nacional a formação no curso superior de enfermagem é direcionada pelas Diretrizes Curriculares Nacionais do Curso de Graduação em Enfermagem, reformulada em 2018, que traça o perfil do enfermeiro, devendo ser generalista, humanista e crítico-reflexivo, com base no saber científico, intelectual e nos princípios éticos, a fim de ser capacitado a atuar no Sistema Único de Saúde (SUS), além de fazer com que a enfermagem seja cada vez mais qualificada (BRASIL, 2018; ESTEVES LSF, et al., 2017).

Para atender esse perfil preconizado ao enfermeiro, o aluno cumprirá os conteúdos curriculares por meio das atividades teóricas, das práticas e do Estágio Curricular Supervisionado (ECS). Os conteúdos curriculares que devem compor a grade de formação são: ciências biológicas e da saúde, ciências humanas, políticas e sociais, ciências exatas e naturais, ciências da enfermagem, educação em saúde e enfermagem, investigação em saúde e enfermagem e temas transversais, sendo importante fazer a ligação desses conteúdos com todo o processo de saúde-doença do indivíduo, da família e da comunidade (BRASIL, 2018).

$\mathrm{Na}$ atividade prática é propiciado ao estudante o desenvolvimento de habilidades técnicas e comportamentais em cenários laboratoriais e reais. No ambiente laboratorial há um professor a cada 10 alunos e, posteriormente, nos locais de atendimento à pacientes e demais cenários, é um professor para seis alunos (BRASIL, 2018).

O curso tem obrigatoriedade de incluir no currículo o período de ECS, implementado oficialmente através das Diretrizes Curriculares Nacionais (DCN), no ano de 2001, sendo imprescindível estar no último ano da graduação, podendo se estender por mais um semestre, e realizado na carga horária mínima de $20 \%$ (vinte por cento) do total de horas que o curso possui (RIGOBELLO JL, et al., 2018). Ele acontece com a preceptoria direta do enfermeiro local e indireta do docente. Quanto aos locais de estágio, sucede nos níveis de atenção primária, secundária, terciária e quaternária (BRASIL, 2018; RAMOS TK, et al., 2018).

No ECS o aluno é o elemento principal na construção de seu conhecimento, ele é inserido na rotina diária do local de estágio, vivenciando as condutas e demais atividades da profissão de Enfermagem, pois através dos diferentes cenários do SUS é oportunizado adquirir conhecimentos e vivências das políticas públicas de saúde, visando a organização do sistema de saúde e o trabalho em equipe multidisciplinar (BRASIL, 2018; NEGREIROS RV e LIMA VCB, 2018; RAMOS TK, et al., 2018). O estágio favorece também a articulação do ensino teórico e prático e possibilita ao acadêmico experiências que o torne um profissional capaz de analisar e responder às situações de forma ideal (ESTEVES LSF, et al., 2017).

Portanto, diante de toda a importância das práticas e estágios para a união do conhecimento teórico com o executar e se aproximar do ambiente de trabalho, acredita-se ser ideal haver mais referências atuais para que os professores e os demais interessados pelo curso de Enfermagem saibam a visão do estudante durante as atividades, sendo, inclusive, pertinente para que haja melhorias no modo de ensinar e na relação alunoprofessor. Portanto, devido a tamanha importância e horas passadas pelo ensino prático, o grupo de estudantes buscou realizar no presente estudo um relato de experiência com o objetivo de referir a experiência de acadêmicas de enfermagem durante as práticas e estágios realizados no decorrer do curso.

\section{RELATO DA EXPERIÊNCIA}

Estudo descritivo, tipo relato de experiência, desenvolvido a partir das vivências de três acadêmicas do curso de enfermagem de uma universidade privada. O curso de enfermagem da instituição é dividido em oito semestres, contemplando aulas teóricas, práticas e o ECS.

A grade curricular do curso de enfermagem é composta por 33 disciplinas e sua estrutura física permite vários ambientes de aprendizagem teórico/prático, sendo salas de aula convencionais; sala Betha, onde por meio do ensino e da tecnologia, com realidade virtual, impressão de peças em tamanhos reais e outros, é possível realizar desde jogos interativos até a inserção do aluno em um cenário real da sua área de estudo. 
Outros recursos muito utilizados para a prática é o laboratório de simulação realística equipado com sistema de som e vidros, que permite a realização de avaliações práticas, e simuladores de alta fidelidade, além de robôs semelhantes ao humano. E a casa simulada, tratando-se do local onde os estudantes participam e assistem à simulações que expõem a realidade diária dos pacientes, gerando aprendizado para 0 atendimento domiciliar. Os alunos ainda dispõem de acesso a biblioteca física e virtual.

A universidade tem articulação com a Secretária Municipal de Saúde (SMS), o qual permite práticas e estágios em diferentes níveis de atenção. Os cenários de Atenção Primária à Saúde (APS) na graduação é a Estratégia Saúde da Família (ESF) e a Unidade Básica de Saúde (UBS). No âmbito de atenção secundária é possível retratar as Unidades de Pronto Atendimento (UPAs) da zona norte e da zona leste como campo de desempenho dos estudantes. Em campo de atuação terciária as atividades ocorreram em um hospital de grande porte e alta complexidade.

Quadro 1 - Características das atividades práticas e ECS de um curso de enfermagem de uma universidade privada, Brasil, 2020.

\begin{tabular}{|c|c|c|c|}
\hline Termo & Nome & $\begin{array}{c}\text { Carga } \\
\text { horária }\end{array}$ & Desempenhos esperados \\
\hline $3^{\circ}$ & Semiologia & 100 & 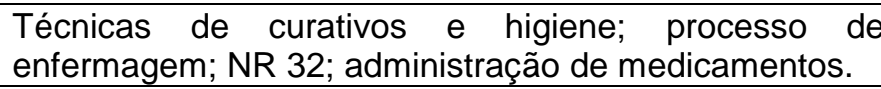 \\
\hline $4^{\circ}$ & $\begin{array}{l}\text { Assistência integrada à } \\
\text { saúde da família }\end{array}$ & 80 & $\begin{array}{l}\text { Visita domiciliar; vacinação; cuidado individual e coletivo; } \\
\text { políticas nacionais de saúde; saúde do homem, da } \\
\text { mulher, da criança e adolescente, do idoso e saúde } \\
\text { mental; educação em saúde. }\end{array}$ \\
\hline \multirow{3}{*}{$5^{\circ}$} & $\begin{array}{l}\text { Assistência de } \\
\text { enfermagem à mulher, } \\
\text { recém-nascido e criança }\end{array}$ & 80 & $\begin{array}{l}\text { Cuidado integral à gestante, puérpera, recém-nascido e } \\
\text { criança; recepção do recém-nascido em sala de parto; } \\
\text { método mãe canguru; educação em saúde materna. }\end{array}$ \\
\hline & $\begin{array}{l}\text { Assistência de } \\
\text { enfermagem clínica }\end{array}$ & 120 & $\begin{array}{l}\text { Cuidado integral ao adulto; processo de enfermagem } \\
\text { reconhecimento de fisiopatologias; análise e aplicação } \\
\text { de curativo, medicamento, oxigenoterapia e terapia } \\
\text { nutricional; cateter nasoenteral e vesical. }\end{array}$ \\
\hline & $\begin{array}{l}\text { Assistência de } \\
\text { enfermagem psiquiátrica }\end{array}$ & 40 & $\begin{array}{l}\text { Cuidado de enfermagem aos pacientes com distúrbios } \\
\text { psiquiátricos e dependência química; avaliação mental; } \\
\text { processo de enfermagem; tratamento farmacológico; } \\
\text { reconhecimento de emergência psiquiátrica; cuidado } \\
\text { familiar. }\end{array}$ \\
\hline \multirow[t]{2}{*}{$6^{\circ}$} & $\begin{array}{l}\text { Assistência de } \\
\text { enfermagem cirúrgica }\end{array}$ & 120 & $\begin{array}{l}\text { Cuidado de enfermagem perioperatório; curativo de } \\
\text { feridas venosas, arteriais e lesões por pressão; avaliação } \\
\text { de drenos; admissão em centro cirúrgico; observação em } \\
\text { sala de cirurgia; paramentação; processo de limpeza e } \\
\text { organização de materiais. }\end{array}$ \\
\hline & $\begin{array}{l}\text { Assistência de } \\
\text { enfermagem em } \\
\text { situações críticas }\end{array}$ & 60 & $\begin{array}{l}\text { Assistência de enfermagem ao paciente grave; drogas } \\
\text { vasoativas; ventilação mecânica; ação em síndromes } \\
\text { coronárianas, politrauma, distúrbio diabéticos e suporte } \\
\text { avançado de vida. }\end{array}$ \\
\hline $7^{\circ}$ & $\begin{array}{l}\text { Estágio curricular } \\
\text { supervisionado I }\end{array}$ & 440 & $\begin{array}{l}\text { Visita domiciliar; reconhecer e atuar no perfil dos } \\
\text { territórios adscritos na ESF; Programa Saúde do Escolar; } \\
\text { Projeto Terapêutico Singular; gestão de recursos; } \\
\text { reunião de equipe e do Conselho Local e Municipal; } \\
\text { espiral construtivista; atividades em situações simuladas; } \\
\text { portfólio de realização. }\end{array}$ \\
\hline $8^{\circ}$ & $\begin{array}{l}\text { Estágio curricular } \\
\text { supervisionado II }\end{array}$ & 440 & $\begin{array}{l}\text { Cuidado integral ao paciente; relacionar o perfil de saúde } \\
\text { dos hospitalizados com a taxa de ocupação; } \\
\text { Classificacaão de Pacientes; Educação em Saúde; gestão } \\
\text { do setor; compreender os tipos de liderança; tomada de } \\
\text { decisão; projeto aplicativo; espiral construtivista. }\end{array}$ \\
\hline
\end{tabular}

Nota: Em todas as práticas a comunicação, atitude ética, responsável, assiduidade e proatividade são desempenhos esperados.

Fonte: Jardim SH, et al., 2020. 
Estar inserido na atenção primária à saúde e hospitalar para as atividades práticas e ECS na graduação permitiu ao discente do curso de enfermagem a aproximação das diferentes dimensões do processo de trabalho do enfermeiro como assistência, pesquisa, ensino e gerência.

$\mathrm{Na}$ assistência esses campos proporcionaram a realização de atividades de extrema importância, sendo elas: anamnese e exame físico, consistindo no momento em que o aluno aprende a questionar o paciente sobre o seu organismo e a examina-lo. E os procedimentos, como passagem de cateter nasoenteral, retirada de acesso venoso periférico e cateterismo vesical de demora. Pôde ainda presenciar uma consulta de enfermagem e o processo de enfermagem.

Na dimensão gerencial o ECS, que acontece no último ano do curso, permitiu ao aluno vivenciar com o enfermeiro supervisor a resolução de conflitos, liderança, elaboração de escalas, notificação de eventos adversos, planejamento em saúde, controle de materiais, trabalho interdisciplinar, gestão de leitos e participação social. Esse estágio exigiu muito do aluno uma comunicação eficaz tanto com o paciente quanto com a equipe para que conseguisse realizar o gerenciamento em enfermagem.

O ensino e a pesquisa estão inseridos no cotidiano do enfermeiro, o acadêmico validou essa afirmação quando observou em campo o enfermeiro ensinando a equipe de enfermagem novos procedimentos e capacitando novos funcionários admitidos no setor. Já na pesquisa o enfermeiro precisa atualizar diariamente seus conhecimentos para praticar uma saúde baseada em evidências.

Alguns desempenhos necessários para a formação do enfermeiro as práticas em campo não conseguem proporcionar ao aluno, por isso, é sanado nos laboratórios de simulação realística. Por exemplo: consulta de enfermagem ao adolescente com distúrbio alimentar, essa é uma situação que dificilmente os alunos conseguem vivenciar no campo de prática, portanto, a simulação acaba sendo uma estratégia de garantir esse desempenho mínimo de atender um adolescente.

As atividades práticas e o ECS proporcionaram ainda às acadêmicas conhecer e entender as Redes de Atenção à Saúde (RAS), aprender a trabalhar em equipe e estar em vários cenários de prática, como campo e laboratórios de simulação. Apesar da importância dessas atividades os estudantes se deparam com a insegurança, distância entre teoria e a prática e a cobrança excessiva e sobrecarga de atividades.

\section{DISCUSSÃO}

O aluno entender as RAS como uma forma de organização dos sistemas de saúde piramidal para o cuidado integral e longitudinal, em que a APS é porta de entrada e reguladora dessa rede, ao qual há uma estrutura com fluxos de comunicação nos diferentes níveis de atenção, e o mesmo estar inserido no meio é de extrema relevância para sua formação, atingindo uma das diretrizes que o prepara para o SUS (PEITER CC, et al., 2019).

O discente sucede as atividades práticas em diversos cenários, ao qual a maioria é vivenciada em unidades de atendimento pelo SUS, sendo uma forma de fazer com que o sistema evolua constantemente. A passagem por diferentes níveis de atenção faz com que o aluno reconheça a continuidade do cuidado, se aproxime das diferentes situações e etapas da vida e das atividades diárias do profissional enfermeiro, tanto em âmbito assistencial quanto na gestão do serviço, podendo alcançar e modificar as ações pertencentes da profissão no futuro (TONHOM SFR, et al., 2016).

O trabalho em equipe é fundamental para realizar uma assistência eficaz e resolutiva aos pacientes, quando a relação da equipe é saudável, e um momento de fornecer ao aluno a possibilidade de aprender a acolher, respeitar, perceber a importância do trabalho em conjunto, entre outros. No entanto, os conflitos também auxiliam na maturidade do estudante, pois percebe que a melhor forma de resolução é através do diálogo (FERNANDES H, et al., 2015; RESTELLATO MTR e DALLACOSTA FM, 2018).

No cargo de enfermeiro há a atribuição de gerência, uma função que se caracteriza por buscar a qualidade do cuidado ao paciente e por oferecer aos profissionais de enfermagem maiores condições de trabalho. Para que o gerenciamento aconteça e possa ser eficaz o enfermeiro precisa realizar com eficiência e sabedoria as suas funções assistenciais, ser líder, ofertar ensino à sua equipe, sendo uma forma de aumentar o conhecimento e melhorar o trabalho, gerenciar os recursos materiais e humanos e fazer a avalição das ações realizadas por si e sob suas organizações (TIRONI MN, et al., 2017). 
O gerenciamento dos recursos para o cuidado precisa ser feito com os princípios da ética e da bioética, para tanto, é essencial que o aluno seja preparado na atividade prática para tal, baseando-se também no sistema de Classificação do paciente para definir o nível de complexidade do cuidado, na cultura organizacional da empresa onde o trabalho está acontecendo e na sistematização da assistência de enfermagem como ferramenta para organização das informações, seguindo com o processo de enfermagem de qualidade (LORENZETTI J, et al., 2014; PÜSCHEL VAA, et al., 2017).

Outra potencialidade importante para o discente são as atividades em laboratório de enfermagem que auxiliam na concepção e reconstrução dos conhecimentos e significados teóricos e práticos para as ações do enfermeiro em ambiente real. O diálogo entre aluno e professor nesse momento é ferramenta importante para o crescimento do mesmo, sendo feito de forma honesta, respeitosa, curiosa, responsável e o deixando pensar criticamente e com autonomia para Ihe ensinar o cuidado com qualidade, não fazendo com que apenas reproduza técnicas (BRITO FMM, et al., 2018).

Entretanto, o aluno se depara com práticas e acontecimentos diferentes ao ensinado em teoria ou em simulação. Os acadêmicos são ensinados sobre o processo de enfermagem com autonomia na tomada de decisão, entretanto, no ambiente real e em determinadas situações não ocorre a interdisciplinaridade, sendo um sistema hierárquico rígido no qual os médicos são os únicos com autonomia efetiva, causando, de certa forma, um detrimento do pensamento, dado que a enfermagem se resulta em algo assistencial sem voz (LORENZETTI J, et al., 2014).

Ainda, quanto a distância da teoria com a prática, a mesma foi encontrada em um estudo realizado com 145 acadêmicos do curso de graduação em enfermagem da Universidade Federal do Triângulo Mineiro no município de Uberaba-MG, onde destacou que o curso necessita de ajustes e adequações para encurtar essa distância, como, dar importância ao papel do docente para proporcionar vivências no campo, haver o domínio de metodologias de ensino que auxiliem no raciocínio clínico, em que há estímulos para a troca de saberes e ao aluno ser o centro do processo de aprender e atualização do corpo docente (GONÇALVES JRL, et al., 2019).

Além disso, o aluno convive com a sobrecarga física e emocional ocasionada pelo ensino letivo, práticas, sociedade e por si mesmo, devido a espera por grandes feitos, baixa capacitação técnica, responsabilidades, a busca por ser um profissional diferente e alcançar as metas do mercado de trabalho e as situações que presencia e precisa agir no processo de vida e morte de pacientes, podendo leva-lo ao estresse diário, síndrome de Burnout, frustrações, inseguranças, dentre outros (FERNANDES MA, et al., 2018; MENEGAZ JC, et al., 2018; PRETO AV, et al., 2018; SILVA LM, et al., 2019).

A experiência do supervisionado gera ao aluno dificuldades emocionais, como o sentimento de insegurança e o medo, tornando esse momento único da graduação algo preocupante, ainda por não ter a presença constante do professor. O mesmo enfrenta constantemente fatores intrínsecos dos setores hospitalares, tais como equipe de enfermagem e médica resistente em aceitar estagiários, fazendo com que não haja acolhimento e sejam inferiorizados, demonstrando indiferença nas suas ações e atitudes que não condizem com a ética e a moral (RESTELATTO MTR e DALLACOSTA FM, 2018).

Diante de toda pesquisa e experiência apresentada, conclui-se que as práticas do curso de enfermagem proporcionam ao acadêmico adquirir hard skills e soft skills, raciocínio clínico e vivência da prática profissional para atuar nos diferentes níveis de atenção à saúde.

\section{REFERÊNCIAS}

1. BRASIL. Ministério da Saúde/Conselho Nacional de Saúde. Resolução n.. 573, de 31 de janeiro de 2018. Diretrizes Curriculares Nacionais do Curso de Graduação em Enfermagem (DCN/ENF). Diário Oficial da União, 2018.

2. BRITO FMM, et al. Nursing laboratory and critical education of nurses: approaches and distances. Revista Brasileira de Enfermagem, 2018; 71(4): 1500-1506.

3. ESTEVES LSF, et al. Supervised internship in undergraduate education in nursing: integrative review. Revista Brasileira de Enfermagem, 2017;71(4): 1740-1750.

4. FERNANDES H, et al. Relacionamento interpessoal no trabalho da equipe multiprofissional de uma unidade de saúde da família. Revista de Pesquisa: Cuidado é Fundamental Online, 2015; 7(1): 1915-1926.

5. FERNANDES MA, et al. Prevalence of anxious and depressive symptoms in college students of a public institution. Revista Brasileira de Enfermagem, 2018; 71(5): 2169-2175. 
6. GONÇALVES JRL, et al. Percepción de los discentes de enfermería del PET GraduaSuS acerca de su formación académica. Revista Família, Ciclos de Vida e Saúde no Contexto Social, 2019; 7(2): 211-219.

7. LORENZETTI J, et al. Work organization in hospital nursing: literature review approach. Text \& Context Nursing, 2014; 23(4): 1104-1112.

8. MENEGAZ JC, et al. Obstacles to the pedagogical encounter between nursing faculty and students in different educational contexts. Educação e Pesquisa, 2018; 44: 1-17.

9. NEGREIROS RV, LIMA VCB. Importância do estágio supervisionado para o acadêmico de Enfermagem no hospital: compartilhando experiências vivenciadas com a equipe de trabalho. Revista da Universidade Vale do Rio Verde, 2018; 16(2): 1-7.

10. PEITER CC, et al. Healthcare networks: trends of knowledge development in Brazil. Escola Anna Nery, 2019; 23(1): 1-10.

11. PRETO AV, et al. Perception of stress in nursing academics. Revista de Enfermagem da Universidade Federal de Pernambuco, 2018; 12(3): 708-715.

12. PÜSCHEL VAA, et al. Nurses in the labor market: professional insertion, competencies and skills. Revista Brasileira de Enfermagem, 2017; 70(6): 1220-1226.

13. RAMOS TK, et al. Estágio curricular supervisionado e a formação do enfermeiro: atividades desenvolvidas. Revista de Enfermagem da Universidade Federal de Santa Maria, 2018; 8(1): 59-71.

14. RESTELATTO MTR, DALLACOSTA FM. Vivências do acadêmico de enfermagem durante o estágio com supervisão indireta. Enfermagem em Foco, 2018; 9(4): 34-38.

15. RIGOBELLO JL, et al. Supervised Curricular Internship and the development of management skills: a perception of graduates, undergraduates, and professors. Escola Anna Nery, 2018; 22(2): 1-9.

16. SILVA LM, et al. Estágio curricular supervisionado: dificuldades e perspectivas vivenciadas por acadêmicos de enfermagem. Revista Eletrônica Acervo Saúde, 2019; 18: 1-10.

17. TIRONI MN, et al. Atribuições e competências de gerentes de enfermagem: pesquisa descritiva exploratória. Revista Online Brazilian Journal of Nursing, 2017; 16(1): 130-139.

18. TONHOM SFR, et al. Nurse's training centred on professional practice: perception of students and professors. Revista Gaúcha de Enfermagem, 2016; 37(4): 1-7.

19. UNIVERSIDADE DO OESTE PAULISTA. Faculdade de Enfermagem Competência: estágio curricular supervisionado I (ECS I) $-7^{\circ}$ termo.

20. UNIVERSIDADE DO OESTE PAULISTA. 2020. In: ESTÁGIO curricular supervisionado II- $8^{\circ}$ termo. Presidente Prudente. Disponível em pdf. Acesso em: 03 out. 2020.

21. XIMENES NETO FRG, et al. Reflections on Brazilian Nursing Education from the regulation of the Unified Health System. Ciência \& Saúde Coletiva, 2020; 25(1): 37-46. 